\title{
Influence of boron doping on the photosensitivity of cubic silicon carbide
}

\author{
V.N. Rodionov ${ }^{1}$, V.Ya. Bratus ${ }^{2}$, S.O. Voronov ${ }^{1}$ \\ ${ }^{I}$ National Technical University of Ukraine "Igor Sikorsky Kyiv Polytechnic Institute” \\ 37, prosp. Peremohy, 03056 Kyiv, Ukraine \\ E-mail:v.rodionov@kpi.ua,s.voronov@kpi.ua \\ ${ }^{2} V$. Lashkaryov Institute of Semiconductor Physics, National Academy of Sciences of Ukraine, \\ 45, prosp. Nauky, 03680 Kyiv, Ukraine \\ E-mail:v_bratus@isp.kiev.ua
}

\begin{abstract}
Photoelectric properties have been studied for 3C-SiC single crystals obtained by thermal decomposition of methyl trichlorosilane with addition of boron in the process of growing or using diffusion into intentionally undoped crystals. Boron-doped samples demonstrate the band of photosensitivity within the range $1.3 \ldots 2.0 \mathrm{eV}$ with the peak near $1.7 \mathrm{eV}$. Doping of 3C-SiC single crystals with B impurity leads to appearance of an efficient recombination center with the thermal activation energy $0.27 \pm 0.02 \mathrm{eV}$ inside the band gap and to widening the spectral sensitivity of the material over the impurity longwave range. Availability of boron results in changing the temperature dependence of photoconductivity from the decay characteristic to the activation one. It will allow expanding the operation range of devices based on $3 \mathrm{C}-\mathrm{SiC}\langle\mathrm{B}\rangle$ up to $500{ }^{\circ} \mathrm{C}$ and above it. In addition, the lux-ampere characteristics becomes linear, i.e., more convenient from the metrological viewpoint. Depending on the type of doping of 3C-SiC $\langle\mathrm{B}\rangle$ samples, pronounced variations of line positions in photoluminescence spectra in near-infrared range are revealed.
\end{abstract}

Keywords: cubic silicon carbide, boron doping, photoconductivity, photoluminescence.

doi: https://doi.org/10.15407/spqeo22.01.92

PACS 61.72.uf, 72.40.+w, 73.50.Pz, 78.55.Hx

Manuscript received 01.02.19; revised version received 23.02.19; accepted for publication 20.02.19; published online 30.03.19.

\section{Introduction}

Cubic and hexagonal polytypes of silicon carbide are considered as a promising material for high-power, highfrequency and high-temperature electronics due to their wide bandgaps, large thermal conductivities, high mobility of carriers and breakdown electric fields [1]. With respect to other polytypes, cubic silicon carbide $3 \mathrm{C}$ $\mathrm{SiC}$ is more stable at lower temperature, and it can be grown below the melting temperature of $\mathrm{Si}\left(1414^{\circ} \mathrm{C}\right)$, which permits its epitaxial growth on $\mathrm{Si}$ substrates. Recent progress in growth of 3C-SiC epitaxial films [2] has opened widespread possibilities for production of high-quality devices and sensors. Application of SiC devices for harsh environment operation necessitates detailed knowledge of impurities, intrinsic and irradiation damage defects and their thermal stability. This paper is devoted to studying the photoconductivity (PC) of borondoped 3C-SiC single crystals within the temperature range $100 \ldots .500 \mathrm{~K}$.
The particular feature of $3 \mathrm{C}-\mathrm{SiC}$ single crystals is their sectorial structure, which is related with difference in the velocities of crystal faces growth: the faces $\{111\}$, $\{211\},\{100\}$ as well as $\{h h l\}$ of the crystallogrophic belt $\langle 110\rangle$ develop the most fast [3]. In the process of doping, there takes place selective adsorption of impurities by different faces, which causes differences in their physical-and-chemical properties. For instance, the boron impurity most efficiently penetrates into the faces $\{111\}$ and $\{211\}$, while that of nitrogen - into the faces $\{h h l\}$. The role of boron, as one of the most important acceptor impurities in $\mathrm{SiC}$, in recombination processes is mainly studied in electrical, luminescent and absorption investigations aimed at hexagonal $4 \mathrm{H}$ and $6 \mathrm{H}$ as well as cubic polytypes [4-8]. At the same time, characteristics of non-radiative recombination channels defining photoelectric properties of $3 \mathrm{C}-\mathrm{SiC}\langle\mathrm{B}\rangle$ are not practically investigated up to date, and it limits their application as various sensors. It is noteworthy that polycrystalline 
3C-SiC with the boron impurity was offered as a material for temperature sensors in the high-temperature range as well as sensors for gas flows [9].

It was earlier shown $[10,11]$ that in $n$-type $3 \mathrm{C}$-SiC crystals the recombination process of non-equilibrium charge carriers is controlled by two types of centers of non-radiative recombination ( $r$ - and $s$-centers) as well as by centers of trapping for major carriers. The $r$-centers define thermal decay of photocurrent with the activation energy of $0.2 \mathrm{eV}$ and capture cross-section for electrons by them $C_{e r}=5 \cdot 10^{-18} \mathrm{~cm}^{2}$, while the latter value for the $s$ centers is $C_{e s}=10^{-16} \mathrm{~cm}^{2}$. The trapping cross-sections for holes by these centers are sharply different $\mathrm{C}_{p r} \gg \mathrm{C}_{\mathrm{ps}}$, and, due to it, the $r$-centers in $n$-type $3 \mathrm{C}$-SiC are the centers of photosensitivity [11]. It was ascertained [12] that the increase in nitrogen concentration results in enhancing the quantum yield for exciton luminescence and in decreasing the lifetime of electrons. In this case, the highest efficiency of photoluminescence is observed in the $\{h h l\}$ growth pyramids with high concentration of uncompensated donors, while the highest photosensitivity - in the $\{111\}$ growth pyramids.

As known, the boron impurity in $\mathrm{SiC}$ can create both shallow and deep levels $[4,5]$. The shallow B-level observed in measurements of deep-level transient spectroscopy (DLTS), Hall effect [4, 5, 13] and electron paramagnetic resonance (EPR) [14] is related to the acceptor centers possessing the activation energy $E_{v}+(0.3 \ldots 0.4) \mathrm{eV}$. The deep B-level pronounced in photoluminescence (PL) and DLTS [4], in absorption [5] and EPR [15] is characterized by creation of a deeper acceptor level. Estimations of the ionization energy for the deep B-level lead to the following values: 0.73 to $1.0 \mathrm{eV}$, when the boron impurity concentration changes from $3.5 \cdot 10^{16}$ up to $1 \cdot 10^{18} \mathrm{~cm}^{-3}$ [7]. The relationship between concentrations of shallow and deep acceptor Bcenters depends on technology of material doping. It is believed that the yellow in $6 \mathrm{H}-\mathrm{SiC}$ [4] and infrared in 3C-SiC [7] PL bands are related with these deep Bcenters. With account of results obtained using EPR and electron-nuclear double resonance (ENDOR), it was drawn the conclusion that boron atoms corresponding to shallow levels substitute silicon atoms in $\mathrm{SiC}$ lattice and create $\mathrm{B}_{\mathrm{Si}^{\mathrm{i}}}$-centers $[14,16]$. In regard to deep B-levels, they are ascribed to the boron - carbon vacancy $\left(\mathrm{B}_{\mathrm{Si}}+\mathrm{V}_{\mathrm{c}}\right)$ complex in accord with the results of ENDOR measurements [17].

The study of PL on undoped epitaxial 3C-SiC films implanted with boron ions found that their spectra, except for typical lines inherent to defects, contain a wide band with the maximum close to $1.6 \mathrm{eV}$ related with boron centers [8]. The estimate of energy for optical ionization of acceptors gives the value $0.7 \mathrm{eV}$ above the valence band top $E_{v}$, which is in good accordance with the results of [7]. The obtained films were offered as photodetectors, but their photoconductive properties remained uncertain.

The aim of this work is to study the influence of the boron acceptor impurity on stationary and relaxation characteristics inherent to the photosensitivity of $3 \mathrm{C}-\mathrm{SiC}$.

\section{Materials and methods}

Studied in this work are photoelectric properties of 3C$\mathrm{SiC}$ single crystals obtained by thermal decomposition of methyl trichlorosilane with addition of boron in the process of growing or using diffusion into intentionally undoped plate-like crystals, separated from which were growth pyramids of the faces $\{111\}$ and $\{211\}$. Before boron diffusion, the crystals were grinded from the side of small face A $\{111\}$ down to the thickness $100 \mu \mathrm{m}$ to reach more uniform distribution of boron. Then the crystals were degreased in $\mathrm{CCl}_{4}$, etched in $\mathrm{HF}+\mathrm{HNO}_{3}$ and washed out in distilled water. In the diffusion process, as a source of doping impurity we used amorphous boron placed on the chamber heater. Diffusion was performed at the temperatures $1700 \ldots 1800^{\circ} \mathrm{C}$ for $20 \ldots 30$ hours in the helium atmosphere. After diffusion, the surface layer of several micrometers was removed. Then, the type of conduction was determined using measurements of thermal e.m.f. or Hall effect. As a result of embedding boron, conduction of the samples was considerably lowered and became of $p$-type. For some samples, we performed measurements of EPR and PL spectra. The availability of boron impurity has been proved by detecting the EPR spectrum of shallow boron in all the samples studied.

In this work, we studied stationary characteristics of photoconductivity: the temperature ones within the range $100 \ldots .500 \mathrm{~K}$, spectral, lux-ampere as well as those of photo-response relaxation.

\section{Results}

Adduced in Fig. 1 are the spectral characteristics of photocurrent measured at the temperature $T=90 \mathrm{~K}$ on specially undoped 3C-SiC sample with $N_{d}-N_{a}=$ $=10^{16} \mathrm{~cm}^{2}$ (curve 1 ), on the sample of the same set as that where diffusion of the boron impurity was performed (curve 2), and on the sample doped with boron in the process of growing (curve 3 ). It is seen from this figure that the spectrum of undoped sample within the impurity range has a weak structure that can be related with availability of background impurities in these crystals [4, 11]. The sample doped in the process of growing demonstrates the band of photosensitivity within the range $1.3 \ldots 2.0 \mathrm{eV}$ with the peak near $1.7 \mathrm{eV}$. The sample doped by diffusion, as compared with the undoped one, also has the considerable photosensitivity within all the impurity range with a weak peak in the same region and approximately the same long-wave boundary for photoconduction.

Essential qualitative changes are observed in the temperature dependence of the concentration of nonequilibrium charge carriers $\Delta n \sim I / \mu$ (Fig. 2), where $I$ is the photocurrent, $\mu-$ mobility of charge carriers. In the initial sample of $n$-type (curve 1 ), within the range of high temperatures $T \geq 170 \mathrm{~K}$ one can observe the photocurrent decay with the activation energy close to $0.2 \mathrm{eV}$. High-temperature boron diffusion or boron 


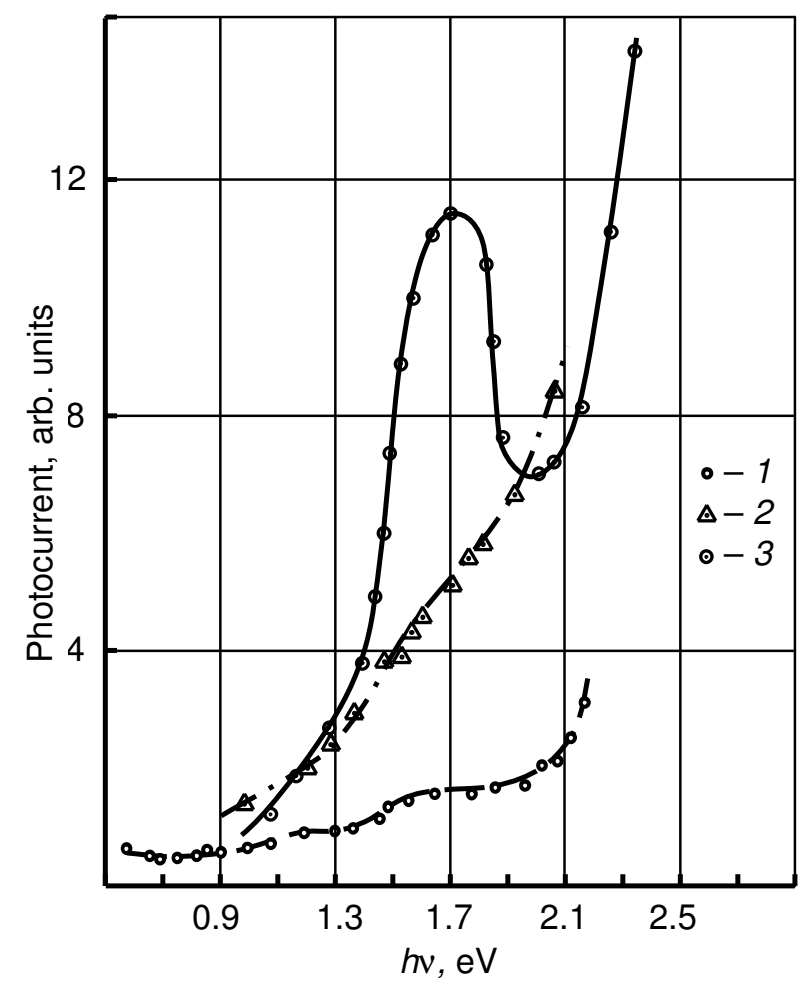

Fig. 1. Spectral dependences of the photocurrent for monocrystalline $3 \mathrm{CSiC}$ samples at $T=90 \mathrm{~K}: 1$ - undoped, 2 diffusion-doped, 3 - doped during growth.

$t, \mathrm{~ms}$

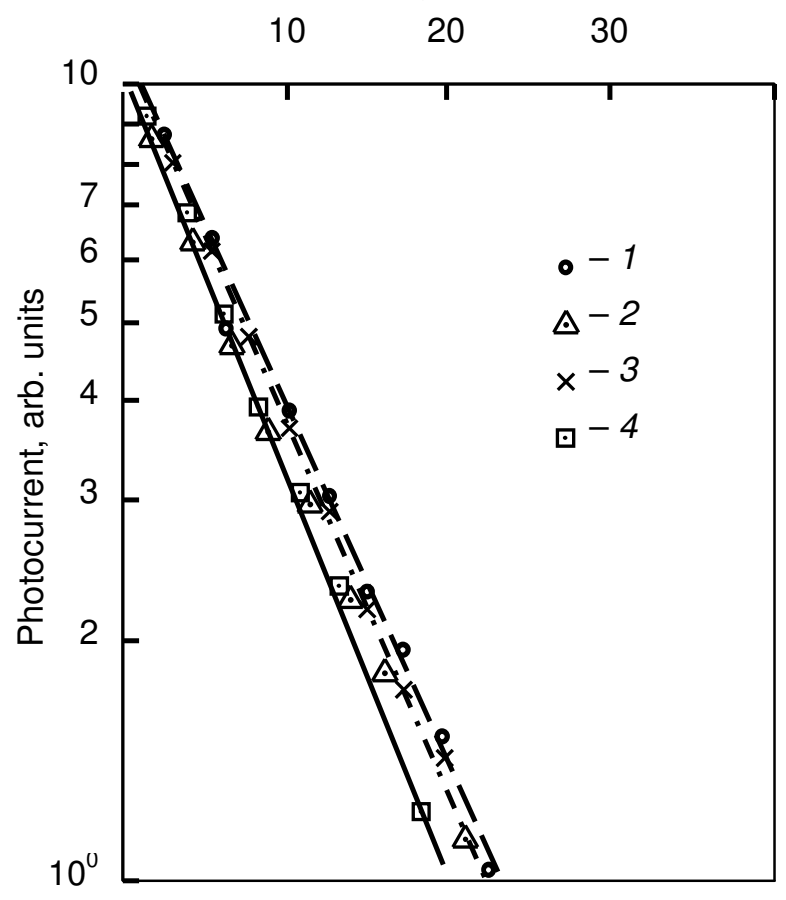

Fig. 3. Photocurrent relaxation under additional illumination $\Phi_{i l l}$ within the range of intrinsic absorption (filter C3C-3): $1-$ without illumination, $\Phi_{i l l}=0,2-\Phi_{i l l} / \Phi_{0}=5,3-\Phi_{i l l} / \Phi_{0}=8$. $\Phi_{0}$ - amplitude of pulsed excitation within the intrinsic band. 4 - illumination within the impurity absorption band $\Phi_{\text {ill }}$ (filter KC-2), $\Phi_{i l l} / \Phi_{0}=1$.

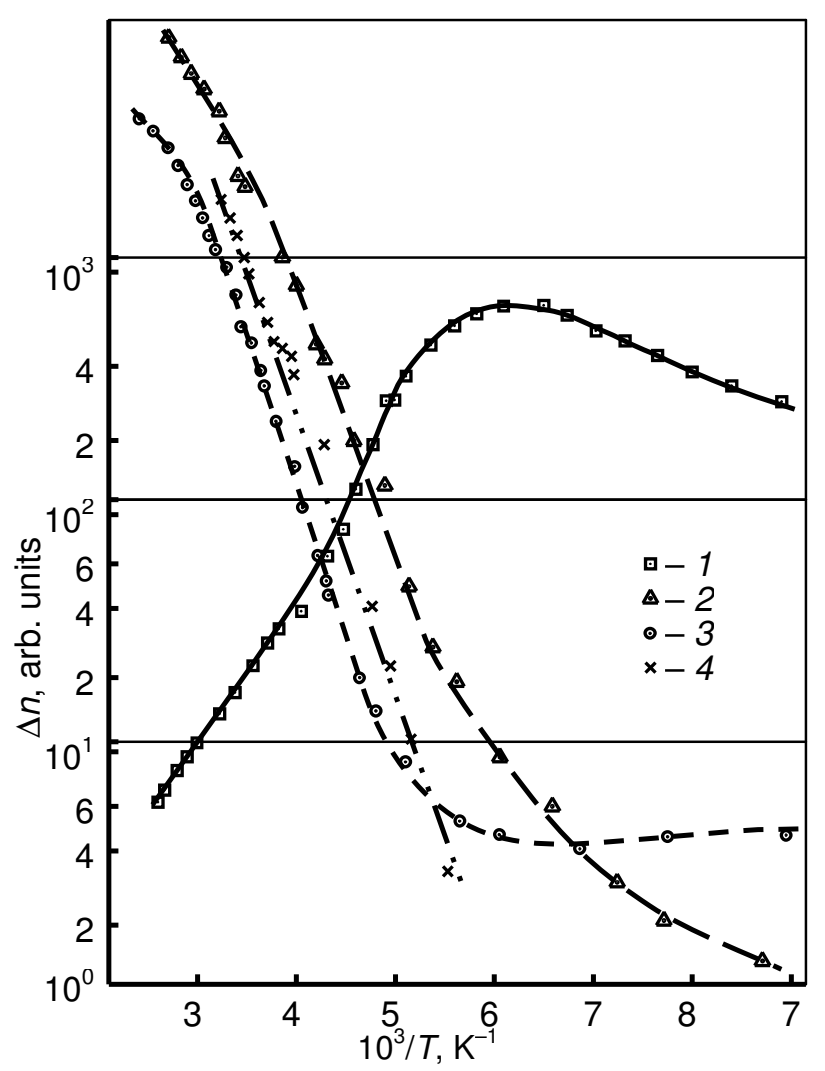

Fig. 2. Temperature dependences of the concentration inherent to non-equilibrium charge carriers, $\Delta n: 1$ - for undoped, 2 - diffusion-doped, and 3 - crystal doped during growth, 4 - dependence of the concentration of equilibrium charge carriers on temperature of a sample doped during growth.

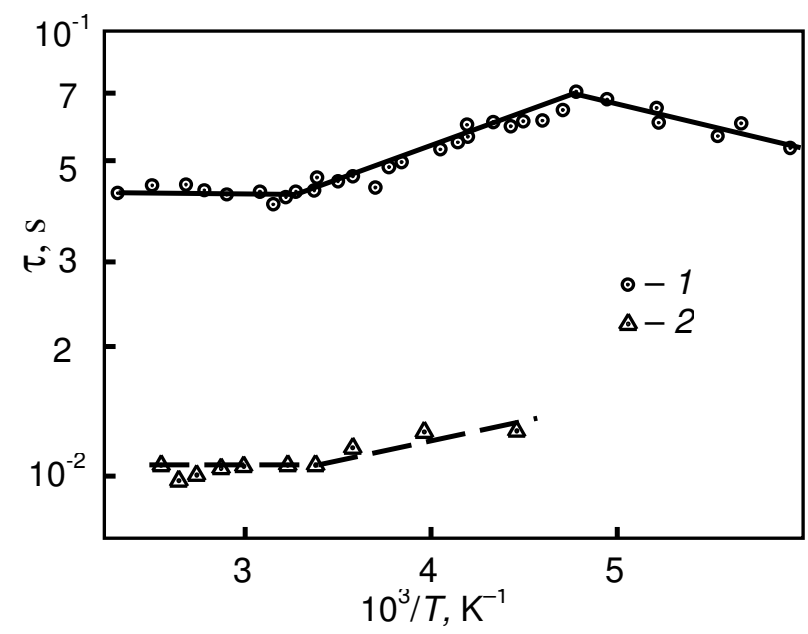

Fig. 4. Temperature dependences of the time constant of the photocurrent decay for the samples doped in the process of growing (1), diffusion (2). 


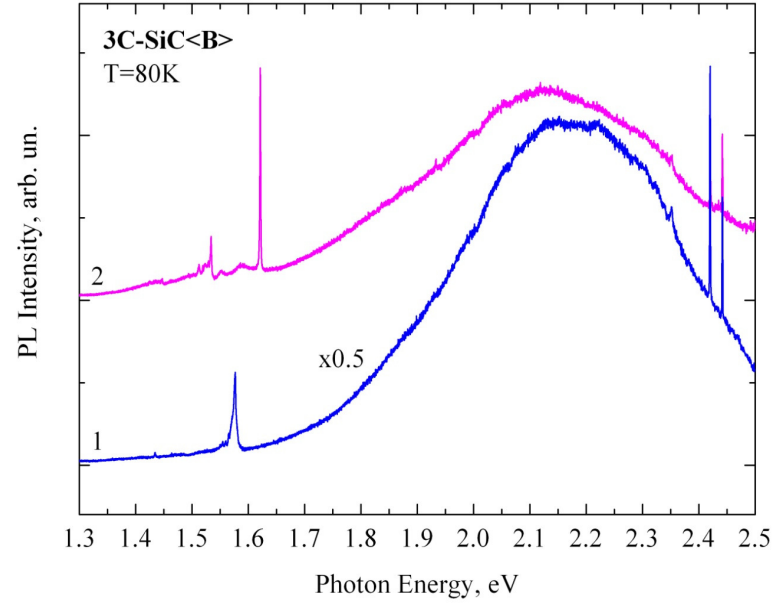

Fig. 5. PL spectra of $3 \mathrm{C}-\mathrm{SiC}\langle\mathrm{B}\rangle$ samples at $80 \mathrm{~K}$ doped with $\mathrm{N}$ and $\mathrm{B}$ during crystal growth (1) and by B diffusion (2). Narrow peaks in the range of $2.41-2.45 \mathrm{eV}$ belong to Raman spectrum.

doping in the process of growing change the character of this temperature dependence (Fig. 2, curves 2 and 3), namely: the photocurrent increases with temperature, and this dependence becomes exponential in a wide temperature range.

The relaxation curves of photocurrent for the samples doped during the process of growing or by diffusion are symmetrical relatively their rise and drop, therefore, they can be approximated with the only exponent having the time constant $\tau=0.01 \ldots 0.05 \mathrm{~s}$ (Fig. 3). In this case, $\tau$ very weakly changes with temperature in the range studied (Fig. 4) both for doped during growth (curve 1) and diffusion (curve 2) samples. Besides, it does not depend on the intensity of additional illumination $\Phi_{i l l}$ with intrinsic or impurity light in regard to the main pulsed illumination $\Phi_{0}$ (Fig. 3).

Lux-ampere characteristics of the studied samples, which were measured in the range of intrinsic absorption at room temperature, indicate that introduction of boron during growth or diffusion results in increasing the exponent in the expression $I \sim \Phi_{0}^{\alpha}$ from $\alpha=0.3$ inherent to the initial undoped sample up to $\alpha=0.8 \ldots 1.0$ for the samples doped by diffusion or during their growth.

\section{Discussion}

As it follows from experimental data, doping of the $3 \mathrm{C}$ $\mathrm{SiC}$ crystals with the boron acceptor impurity by using both above methods leads to the increase in the material resistivity, changes in the shape of PC spectrum, in the character of photocurrent temperature dependence, its kinetics, as well as changes in the exponent of luxampere characteristics. PC measurements reveal a new band with the long-wave edge near $1.3 \mathrm{eV}$ and the maximum close to $1.7 \mathrm{eV}$ (Fig. 1), which is in accord with the data of absorption measurements [8]. As compared with the initial samples, the ones doped by diffusion show an enhanced photosensitivity inside a wider range up to the fundamental edge of material (curve 2). As it follows from this figure, PC spectra of the doped samples differ: in the case of diffusion doping, the impurity band is considerably wider, which can be caused by creation of impurity-defect complexes in the process of $\mathrm{B}$ introduction [18]. It is also noteworthy that the samples doped by diffusion demonstrate the emission band related with $\mathrm{B}$ impurity in $6 \mathrm{H}$ polytype [19], which is not observed in the samples doped during their growth [18].

As it follows from Fig. 2 (curve 2), temperature activation of samples doped by diffusion is related with the center possessing the energy of thermal ionization $0.27 \pm 0.02 \mathrm{eV}$. Its appearance is caused by boron introduction, since the samples doped in the process of growing (curve 3) have approximately the same slope. Taking into account that the temperature dependence of carrier dark concentration after boron doping has approximately the same look (curve 4), one can assume that this center of acceptor type defines also PC spectral characteristics of $3 \mathrm{C}-\mathrm{SiC}$ doped with boron. Like to the centers in $\mathrm{CdS}$ [20], it is fully or partly radiationless. Activation of the photocurrent in $3 \mathrm{C}$-SiC of $p$-type can be explained by the decrease in filling recombination centers with holes, when temperature is increased, as well as with increasing the stationary lifetime of holes in the temperature range $T \geq 150 \mathrm{~K}$.

It follows from Fig. 3 that relaxation of photocurrent is defined by one exponent with the time constant weakly depending on temperature in a wide range (Fig. 4) and on additional illumination with light from the fundamental edge or from the impurity absorption range as well. This fact along with the linear lux-ampere characteristic means that photoconductivity in the B-doped samples in our experimental conditions has monopolar character, and the process of nonequilibrium carriers recombination is controlled by the only efficient deep center. To identify it, we need some additional investigations.

It has been found earlier for $6 \mathrm{H}-\mathrm{SiC}$ that B-related PL band position and intensity vary in dependence on the type of doping [6]. Our verification with PL of $3 \mathrm{C}-\mathrm{SiC}\langle\mathrm{B}\rangle$ samples prepared by doping of crystals simultaneously with $\mathrm{N}$ and $\mathrm{B}$ during crystal growth as well as the samples prepared using B diffusion into unintentionally $\mathrm{N}$-doped crystals reveals the same feature. Fig. 5 shows PL spectra of both samples at $80 \mathrm{~K}$ excited by the 488-nm line of an $\mathrm{Ar}^{+}$-ion laser with an interferential filter. Both of these spectra display broad emission band centered at about $2.15 \mathrm{eV}$ and sets of narrow peaks in near-infrared region. By contrast to H. Kuwabara et al. [7], who observed a broad band for nitrogen donor - boron acceptor pair recombination with zero-phonon line (ZPL) at $1.640 \mathrm{eV}$ in crystals grown from the melt, the positions of ZPL equal to 1.621 and $1.577 \mathrm{eV}$ for $3 \mathrm{C}-\mathrm{SiC}\langle\mathrm{B}\rangle$ samples doped from vapor and by diffusion, respectively. Pronounced variation of position and general view of PL spectra for $3 \mathrm{C}-\mathrm{SiC}\langle\mathrm{B}\rangle$ samples, depending on the type of doping, needs further investigations. 


\section{Conclusions}

- $\quad$ Doping of $3 \mathrm{C}-\mathrm{SiC}$ single crystals with the acceptor $\mathrm{B}$ impurity during growing or diffusion leads to appearance of the efficient recombination center with the thermal activation energy $0.27 \pm 0.02 \mathrm{eV}$ inside the material band gap and to widening the spectral sensitivity of this material over the impurity long-wave range.

- Doping with boron results in changing the temperature dependence of photoconductivity from the decay characteristic to the activation one. It will allow expanding the operation range of devices based on $3 \mathrm{C}-\mathrm{SiC}\langle\mathrm{B}\rangle$ up to $500{ }^{\circ} \mathrm{C}$ and above it.

- After embedding boron, the lux-ampere characteristic becomes linear, i.e., more convenient from the metrological viewpoint.

\section{Acknowledgments}

The authors are grateful to Dr. O. Kolomys and Prof. V. Strelchuk for assistance in PL characterizations.

\section{References}

1. Silicon Carbide. V. 2: Power Devices and Sensors. P. Friedrichs, T. Kimoto, L. Ley, G. Pensl (eds.). Wiley-VCH Verlag GmbH, Weinheim, 2011.

2. Fundamentals of Silicon Carbide Technology: Growth, Characterization, Devices and Applications. T. Kimoto and J.A. Cooper (eds.). Wiley-IEEE Press, 2014.

3. Gorin S.N. and Ivanova L.M. Cubic silicon carbide (3C-SiC): Structure and properties of single crystals grown by thermal decomposition of methyl trichlorosilane in hydrogen. phys. status solidi $(b)$. 1997. 202. P. 221-245; https://doi.org/10.1002/15213951(199707)202:1<221::AID-PSSB221> 3.0.CO;2-L.

4. Suttrop W., Pensl G., Lanig P. Boron-related deep centers in 6H-SiC. Appl. Phys. A. 1990. 51. P. 231237; https://doi.org/10.1134/1.1187657.

5. Lebedev A.A. Deep level centers in silicon carbide: A review. Semiconductors. 1999. 33. P. 107-130.

6. Hagen S.H., Kemenade A.W.C. On the role of boron in the luminescence of silicon carbide doped with nitrogen and boron. physica status solidi (a). 1976. 33, No 1. P. 97-105. DOI: $10.1002 /$ pssa.2210330109.

7. Kuwabara H., Yamada S. Free-to-bound transition in $\beta$-SiC doped with boron. physica status solidi (a). 1975. 30. P. 739-746; https://doi.org/10.1002/pssa.2210300234.

8. M. Syväjärvi, Ma Quanbao, Jokubavicius V., Galeckas A. et al. Cubic silicon carbide as a potential photovoltaic material. Solar Energy Materials \& Solar Cells. 2016. 145. P. 104-108; doi: 10.1016/j.solmat.2015.08.029.

9. Bubulis A., Voronov S.A., Genkin A.M., Bratus T.I., Rodionov V.N. Thermoanemometery based on polycrystalline silicon carbide cubic modification. Bulletin of National Technical University of Ukraine "Kyiv Politechnic Institute, Series INSTRUMENT MAKING. 2016. 52, No 2. P. 42-47.

10. Altaisky Y.M., Pletjushkin A.A., Rodionov V.N. The temperature dependence of the photocurrent in cubic silicon carbide. Ukr. J. Phys. 1985. 30, No 9. P. 1417-1420.

11. Altaisky Y.M., Rodionov V.N. About main parameters of the recombination centers in cubic silicon carbide. Ukr. J. Phys. 1985. 30, No 10. P. 1512-1515.

12. Rodionov V.N., Bratus' V.Ya. Influence of nitrogen impurity on radiative and nonradiative recombination in cubic silicon carbide. Ukr. J. Phys. 2001. 46. P. 979-984.

13. O.V. Aleksandrov, E.N. Mokhov. Model of boron diffusion from gas phase in silicon carbide. Semiconductors. 2011. 45, No 6. P. 705-712. DOI: 10.1134/S1063782611060029.

14. Baran N.P., V Bratus'.Ya., Bugai A.A., Vikhnin V.S., Klimov A.A., Maksimenko V.M., Petrenko T.L., Romanenko V.V. Electron spin resonance of boron in cubic SiC: manifestation of the Jahn-Teller effect. Phys. Solid State. 1993. 35, No 11. P. 15441548.

15. Baranov P.G., Mokhov E.N. Electron paramagnetic resonance of deep boron in silicon carbide. Semicond. Sci. Technol. 1996. 11. P. 489-494; https://doi.org/10.1088/0268-1242/11/4/005.

16. Petrenko T.T., Petrenko T.L. Density functional theory study of the shallow boron impurity in 3C$\mathrm{SiC}$ and comparison with experimental data. Phys. Rev. B. 2016. 93. P. 165203; https://doi.org/10.1103/PhysRevB.93.165203.

17. van Duijn-Arnold A., Ikoma T., Poluektov O.G., Baranov P.G., Mokhov E.N., Schmidt J. Electronic structure of the deep boron acceptor in boron-doped 6H-SiC. Phys. Rev. B. 1998. 57. P. 1607-1619.

18. Ballandovich V.S., Mokhov E.N. Annealing of deep boron centers in silicon carbide. Semiconductors. 2002. 36. P. 160-166.

19. Violin E.E. and Kholuyanov G.F. Extraction of carriers by the field of the $p-n$ junction and mechanism of electroluminescence in SiC. Sov. Phys. Solid State. 1966. 8. P. 2716-2718.

20. Lyubchenko A.V., Sheinkman M.K. The temperature quenching of the photocurrent and photoluminescence in wideband semiconductors. Ukr. J. Phys. 1973. 18, No 2. P. 291-299. 


\section{Authors and CV}

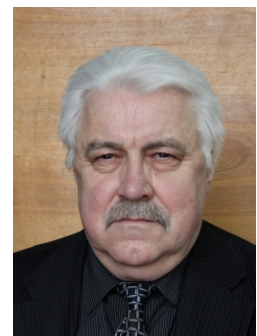

Voronov S.A. Professor, Doctor of Technical Sciences, Chief of Department of Applied Physics, I. Sikorskyi National Technical University of Ukraine "Kyiv Polytechnic Institute". The area of scientific interest of Prof. S.A. Voronov includes optoelectronics and infrared techniques.

I. Sikorskyi National Technical University of Ukraine "Kyiv Polytechnic Institute"

E-mail s.voronov@ kpi.ua ORCID ID 0000-0002-0053-0381

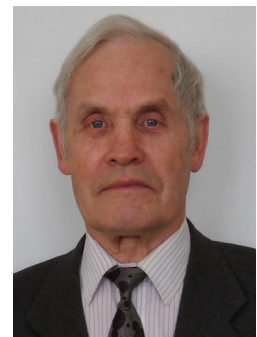

Rodionov V.N. Senior Researcher, Doctor of Phil., I. Sikorskyi National Technical University of Ukraine "Kyiv Polytechnic Institute". The area of scientific interest of Researcher V.N. Rodionov includes photoelectrical and luminescent processes in solid state.

I. Sikorskyi National Technical University of Ukraine "Kyiv Polytechnic Institute"

E-mail v.rodionov@kpi.ua ORCID ID 0000-0001-6300-4840

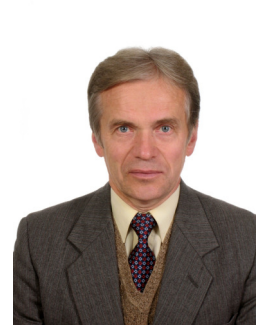

Bratus' V.Ya. Doctor of Sciences in Physics and Mathematics, Acting Head of the Laboratory of Radiospectroscopy in the Department of Optics and Spectroscopy, V. Lashkaryov Institute of Semiconductor Physics, NAS of Ukraine. The area of scientific interests of Dr. Bratus' includes determination of intrinsic and extrinsic defects in semiconductors and solid state by EPR, ENDOR and photoluminescence.

V. Lashkaryov Institute of Semiconductor Physics, National Academy of Sciences of Ukraine

E-mail: v_bratus@isp.kiev.ua 